\title{
Serum Lipocalin 2 Levels in Acute Exercised Prediabetic Rat Model in Comparison to Normal Healthy Control
}

\author{
Rania Reafaat Abdelkader Atia ${ }^{*}$, Khaled Abdelfattah Abdelhamid Abulfadle \\ Physiology department, Faculty of Medicine, Zagazig University, Zagazig, Egypt \\ Email address: \\ raniareafaat777@gmail.com (R. R. A. Atia),khafadle@gmail.com (K. A. A. Abulfadle) \\ To cite this article: \\ Rania Reafaat Abdelkader Atia, Khaled Abdelfattah Abdelhamid Abulfadle. Serum Lipocalin 2 Levels in Acute Exercised Prediabetic Rat \\ Model in Comparison to Normal Healthy Control. American Journal of Clinical and Experimental Medicine. \\ Vol. 3, No. 3, 2015, pp. 105-109. doi: 10.11648/j.ajcem.20150303.16
}

\begin{abstract}
Background: Excess caloric intake expands adipose tissue resulting in a higher risk of metabolic disease. Lipocalin 2 (LCN2) is an adipokine having a role in innate immunity and energy metabolism. It is an acute phase protein that was recently correlated with potential effects on obesity and inflammation. The reaction of this protein to progressive exercise in animals has limited studies. Exercise encourages weight loss and improves insulin sympathy. However, the molecular mechanisms mediating its beneficial effects are not fully understood. Obesity associates with increased production of inflammatory cytokines, which in turn, subsidizes to systemic insulin resistance. LCN2 has been shown to have a significant association with body mass index (BMI). However, only few of the LCN2-related studies have investigated whether LCN2 constitutes a causal factor of the metabolic conditions in animals. One of the best strategies to prevent obesity and its associated inflammation is participation in regular physical activity. Objective: This study was designed to test the hypothesis that exercise mitigates this inflammatory response, thereby improving insulin sensitivity, we tested whether there is an insulinsensitizing effect of exercise is associated with related changes in circulating LCN2 levels in rats. Design: A total number of 24 adult healthy male albino rats were used. Rats were divided into three groups: group I (control, C), group II (model of prediabetic obese rats without exercise, PD) and group III (model of pre-diabetic obese rats with acute voluntary exercise, PDE). Results: This study revealed that after a 2 days acute exercise program, insulin sensitivity was improved, as indicated by a significant decrease in serum insulin (1.98 \pm 0.04$)$, serum glucose (121.75 \pm 2.85$)$ and Homeostasis Model Assessment for Insulin Resistance Index (HOMA-IR) $(0.59 \pm 0.02)$ in PDE in comparison to corresponding values in PD group (3.94 \pm 0.08$)$, $(159 \pm 1.49)$ and $(1.44 \pm 0.11)$ respectively. Also, Serum LCN2 concentrations were significantly decreased (62.88 \pm 1.79$)$ in PDE group in comparison to PD group (94.75 \pm 3.36$)$. Conclusion: We concluded that exercise has an insulin sensitizing effect in the pre-diabetic stage and its mechanism could involve a reduction in LCN2 levels in exercise-trained group.
\end{abstract}

Keywords: Insulin Resistance, Lipocalin 2, Glucose, Adipocytes, Exercise, HOMA-IR, Rats

\section{Introduction}

Lipocalin 2 (LCN2), an adipokine protein abundantly secreted by adipocytes, has a role in metabolism [1]. Zhang, $\mathrm{Wu}$ [2] stated that diet-induced obesity increased adipose tissue LCN2 expression. Also Guo, Jin [3] said that LCN2 has a role in obesity and insulin resistance as demonstrated by studies on LCN2 knockout mice. Moreover, Zhang, Foncea [4] found that LCN2 secretion is stimulated by insulin through NFkB signaling activation and glucose metabolism. Diabetes is a chronic metabolic disorder with a rapidly growing prevalence and is predisposed by a sedentary life and an unhealthy diet [5]. Individuals having an active physical life did not suffer from impaired glucoses tolerance or non-insulin dependent diabetes [6]. Regular body exercise was found to be important in treatment of type 2 diabetes mellitus (T2DM) as it increased insulin sensitivity [5]. Adipose tissue has a dynamic role as a lipidstoring and endocrine organ that synthesizes a number of biologically active compounds that regulate energy metabolism, a state of equilibrium to body weight, inflammation, and insulin resistance [7]. White adipose tissue secretes and expresses receptors for many hormones, growth factors and cytokines [8]. Unbalanced production of 
certain adipocytokines is considered as a cause of lifestylerelated diseases as diabetes mellitus [9]. Thus, reduction of body fat (especially visceral) is of value in prevention of such diseases [10]. Adipose tissue composition is compromised in response to metabolic stress, excessive caloric intake, and aging. Specifically, adipose tissue undergoes infiltration of proinflammatory macrophages and $\mathrm{T}$ cells, adipocyte hyperplasia and hypertrophy, and extracellular matrix remodeling during obesity, leading to adipose tissue dysfunction and insulin resistance [11]. Adipose tissue remodeling responds to alterations in the nutrition state to be increased in obese persons [11]. Also, muscular exercise affects energy balance and stimulates some changes in body metabolism as it shares in regulation of blood glucose level through stimulation of hypothalamicpituitary-adrenal axis and control of the secretory function of the adipose tissue [12]. On the other hand, Guo, Bazuine [13] found an increase in body fat mass and insulin resistance in LCN2-deficient mice with high fat diet. For this reason, this study designated to explore the effect of acute exercise protocol on a LCN2 protein and metabolic syndrome markers of insulin resistance in normal and prediabetic model of male albino rats.

\section{Material and Methods}

\subsection{Animals}

The pre-diabetic rat model is produced by developing obesity to non-obese non diabetic rats when fed on high energy diet instead of its usual low energy vegetable diet. A total number of twenty four male albino rats (weight range, 150-200 g) were purchased from the animal house of Zagazig University. For acclimatization, rats were supplied with a standard chow diet and tap water for 5 days. Rats were put, individually in cages for better measuring of intake of food and to decrease rat movements, in a 12:12 hour lightdark cycle and hygienically controlled room [14]. Then, rats were subdivided into 3 groups: Control (C) group, for 4 weeks, rats fed on usual low energy vegetable diet and for drinking, they receive tap water. Pre-diabetic (PD) group, for 4 weeks, rats fed on a high-fat diet (HFD i.e. $60 \%$ calories from fat, $20 \%$ calories from protein, $20 \%$ calories from carbohydrate) [15] and for drinking, they receive tap water. Pre-diabetic with exercise (PDE) group, for 4 weeks, rats fed on high-fat diet and for drinking, they receive tap water, then, they have an exercise protocol.

\subsection{Exercise Protocol}

For 2 days, rats of PDE group were exposed to acute exercise through swimming. In plastic barrels of $45 \mathrm{~cm}$ in diameter filled with water (at $\sim 32^{\circ} \mathrm{C}$ ) to a depth of $60 \mathrm{~cm}$, rats swam in groups of three, for two bouts each was 3 hours with a 45 min rest separating them [16]. Just after the end of exercise, the rats were sacrificed under ether anesthesia by decapitation.

\subsection{Measurement of Serum Parameters}

Blood samples were collected from all groups. Sera were separated and fasting serum glucose was measured using an automated analyzer (YSI 2300; YSI Life Sciences, Yellow Springs, $\mathrm{OH}$ ) via the glucose oxidase method. Insulin was measured using commercial ELISA kits (R\&D Systems, Minneapolis, MN) and LCN2 levels were measured using Lipocalin-2 (NGAL) Rat ELISA Kit (ab119602) and insulin resistance (IR) was evaluated by calculating the homeostasis model assessment-IR (HOMA-IR) index. This index is calculated as follows:

$$
\begin{aligned}
\text { HOMA-IR }= & {[\text { fasting glucose }(\mathrm{mg} / \mathrm{dL}) \times \text { fasting insulin }} \\
& (\mu \mathrm{IU} / \mathrm{mL})] \times(405)-1[17] .
\end{aligned}
$$

The value of this index is interpreted as follows: when the value of HOMA-IR increases, IR augments, which means that insulin sensitivity decreases [18]. In order to decide whether the treatments had an effect on IR, the value of the HOMA-IR index was compared among the groups.

\subsection{Statistical Analysis}

Data were analyzed by using SPSS for windows package version (21) and described as Mean \pm SEM. One way ANOVA and Tukey HSD post hoc test for multiple group comparison were used for calculating the statistical significance. Significance was set at $\mathrm{P} \leq 0.05$.

\section{Results}

Table-1 and figure- 1 showed the effect of acute exercise on Insulin $(\mu \mathrm{IU} / \mathrm{mL})$ among different groups. There was a significant increase in serum insulin in the PD group (3.94 \pm 0.08$)$ in comparison to the $C$ group $(1.99 \pm 0.06)$, but, there was a significant decrease in serum insulin in the PDE group (1.98 \pm 0.04$)$ in comparison to the $\mathrm{PD}$ group (3.94 \pm 0.08$)$. Also, table- 1 and figure- 2 showed the effect of acute exercise on glucose $(\mathrm{mg} / \mathrm{dL})$ among different groups. There was a significant increase in serum glucose in the PD group $(159 \pm 1.49)$ in comparison to the $\mathrm{C}$ group (125.75 \pm 2.71$)$, but, there was a significant decrease in serum glucose in the PDE group (121.75 \pm 2.85$)$ in comparison to the PD group (159 \pm 1.49$)$. Moreover, table-1 and figure-3 showed the effect of acute exercise on HOMA-IR among different groups. There was a significant increase in HOMAIR in the PD group $(1.44 \pm 0.11)$ in comparison to the $C$ group $(0.62 \pm 0.02)$, but, there was a significant decrease in HOMAIR in the PDE group $(0.59 \pm 0.02)$ in comparison to the PD group (1.44 \pm 0.11$)$. Furthermore, table- 1 and figure- 4 showed the effect of acute exercise on HOMA-IR among different groups. There was a significant increase in serum LCN2 in the PD group (94.75 \pm 3.36$)$ in comparison to the $\mathrm{C}$ group $(53.38 \pm 2.18)$, but, there was a significant decrease in serum LCN2 in the PDE group $(62.88 \pm 1.79)$ in comparison to the PD group (94.75 \pm 3.36$)$. 
Table 1. Effect of acute exercise on serum insulin ( $\mu I U / m L)$, serum glucose (mg/dL), HOMA-IR and serum LCN2 (in ng/ml) among different groups.

\begin{tabular}{llll}
\hline & Control $(C)$ & Prediabetic (PD) & Prediabetic with exercise (PDE) \\
\hline Insulin $(\mu \mathrm{IU} / \mathrm{mL})$ & $1.99 \pm 0.06$ & $3.94 \pm 0.08^{\mathrm{a}}$ & $1.98 \pm 0.04^{\mathrm{b}, \mathrm{c}}$ \\
Glucose $(\mathrm{mg} / \mathrm{dL})$ & $125.75 \pm 2.71$ & $159 \pm 1.49^{\mathrm{a}}$ & $121.75 \pm 2.85^{\mathrm{b}, \mathrm{c}}$ \\
HOMA-IR & $0.62 \pm 0.02$ & $1.44 \pm 0.11^{\mathrm{a}}$ & $0.59 \pm 0.02^{\mathrm{b}, \mathrm{c}}$ \\
LCN2 $(\mathrm{ng} / \mathrm{mL})$ & $53.38 \pm 2.18$ & $94.75 \pm 3.36^{\mathrm{a}}$ & $62.88 \pm 1.79^{\mathrm{c}, \mathrm{d}}$ \\
\hline
\end{tabular}

Data was expressed as Mean \pm SEM. ${ }^{a} \mathrm{P}<0.001,{ }^{\mathrm{d}} \mathrm{P}<0.05$ and ${ }^{\mathrm{b}} \mathrm{P}>0.05$ in comparison to the $\mathrm{C}$ group. ${ }^{\mathrm{c}} \mathrm{P}<0.001$ in comparison to $\mathrm{PD}$ group.

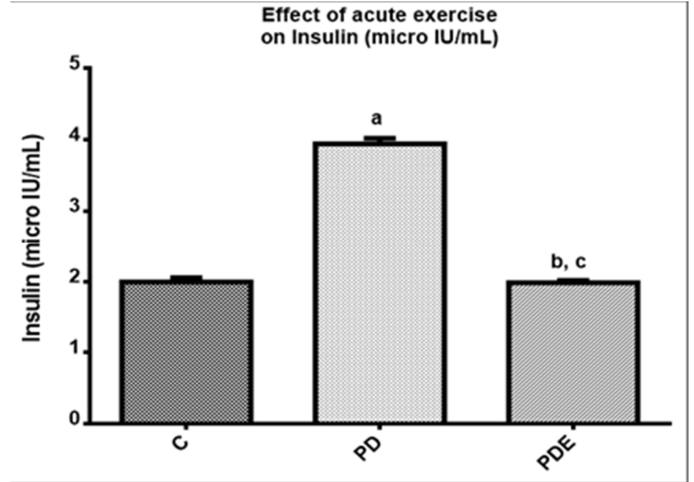

Data was expressed as Mean \pm SEM. ${ }^{a} \mathrm{P}<0.001$ and ${ }^{b} \mathrm{P}>0.05$ in comparison to the $\mathrm{C}$ group. ${ }^{\mathrm{c}} \mathrm{P}<0.001$ in comparison to $\mathrm{PD}$ group.

Figure 1. Effect of acute exercise on serum insulin $(\mu \mathrm{IU} / \mathrm{mL})$ among different groups.

\section{Effect of acute exercise on glucose $(\mathrm{mg} / \mathrm{dL})$}

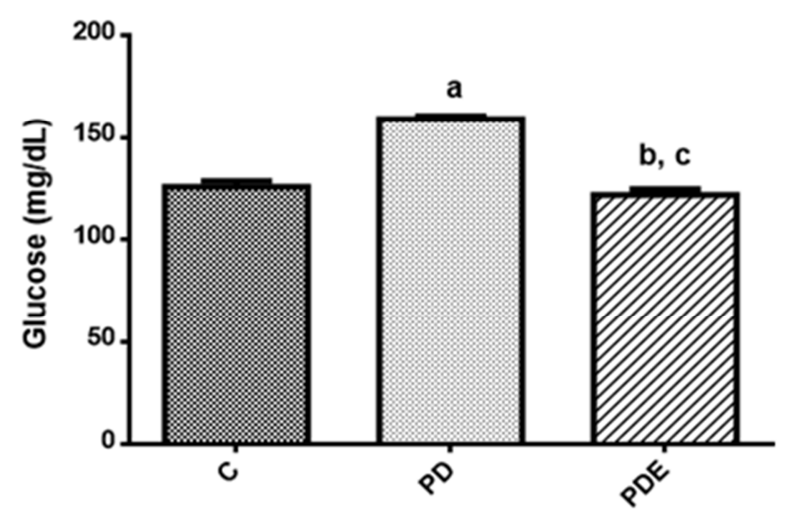

Data was expressed as Mean \pm SEM. ${ }^{a} \mathrm{P}<0.001$ and ${ }^{\mathrm{b}} \mathrm{P}>0.05$ in comparison to the $\mathrm{C}$ group. ${ }^{\mathrm{C}} \mathrm{P}<0.001$ in comparison to $\mathrm{PD}$ group.

Figure 2. Effect of acute exercise on serum glucose ( $\mathrm{mg} / \mathrm{dL}$ ) among different groups.

\section{Discussion}

The results of this study showed that there is a significant increase in serum LCN2, serum glucose, serum insulin and HOMA-IR in the PD group in comparison to the $\mathrm{C}$ group. These results indicates that obesity causes increase in both serum LCN2 and insulin resistance. Our results are supported by Yan, Yang [1] who stated that LCN2 is produced and secreted by mature adipocytes and its expression is significantly increased during adipogenesis as evidenced their studies on ob/ob and db/db mice. Also, Catalan, Gomez-

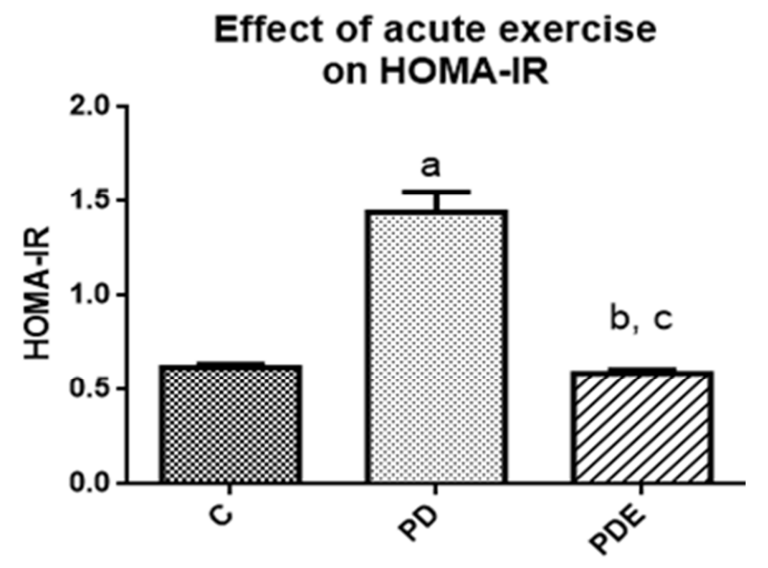

Data was expressed as Mean \pm SEM. ${ }^{\mathrm{a}} \mathrm{P}<0.001$ and ${ }^{\mathrm{b}} \mathrm{P}>0.05$ in comparison to the $\mathrm{C}$ group. ${ }^{\mathrm{c}} \mathrm{P}<0.001$ in comparison to $\mathrm{PD}$ group.

Figure 3. Effect of acute exercise on HOMA-IR among different groups.

\section{Effect of acute exercise on LCN2 (ng/ml)}

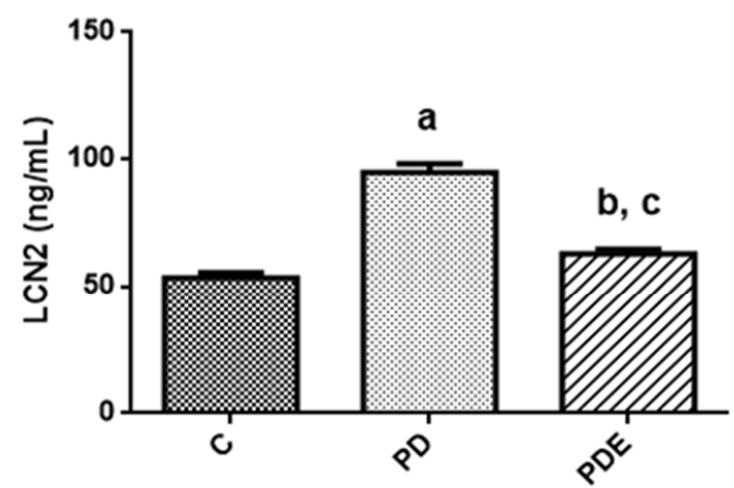

Data was expressed as Mean \pm SEM. ${ }^{a} \mathrm{P}<0.001$ and ${ }^{\mathrm{b}} \mathrm{P}<0.05$ in comparison to the $\mathrm{C}$ group. ${ }^{\mathrm{c}} \mathrm{P}<0.001$ in comparison to $\mathrm{PD}$ group.

Figure 4. Effect of acute exercise on serum LCN2 (in $\mathrm{ng} / \mathrm{ml}$ ) among different groups.

Ambrosi [19] found that circulating LCN2 is positively related with adiposity, blood triglyceride, blood glucose and insulin resistance, and negatively associated with highdensity lipoprotein cholesterol. Moreover, Sommer, Weise [20] studies have shown that some inducers of insulin resistance and glucocorticoids up-regulated LCN2 expression while, the insulin sensitizer thiazolidinedione inhibited its expression. Furthermore, Yan, Yang [1] had been demonstrated that LCN2 expression is increased in obesity and insulin resistance, the results which were further confirmed by their study on shRNA-mediated knockdown of 
LCN2 which resulted in improvement of insulin sensitivity and insulin-stimulated glucose uptake in adipocytes. Also, our results declared that there is a significant decrease in serum LCN2, serum glucose, serum insulin and HOMA-IR in the PDE group in comparison to the PD group which indicated that acute exercise decreased serum LCN2 and at the same time increased insulin sensitivity. Thus, it is clear that there is a link between serum LCN2 and insulin sensitivity which explains the insulin sensitizing effect of exercise which may be through decreasing serum LCN2. These results are supported by Luciano, Carneiro [21], Aoi, Ichiishi [22], Ropelle, Pauli [16], Flores, Fernandes [23], Pauli, Ropelle [24], Peres, de Moraes [25] and Ropelle, Pauli [26] who stated that acute exercise training, can improve insulin sensitivity of obese rats. Also, our results were supported by Law, Xu [27] who found that LCN2-deficient mice were protected from obesity-related insulin resistance.

On the contrary, a study from Chen's lab reported that LCN2 deficiency results in impairment of insulin sensitivity [3]. Also, Rosen's lab published a paper that showed that LCN2 knockout does not substantially affect obesity-induced insulin resistance [28]. Moreover, Guo, Bazuine [29] stated that LCN2 deficiency enhances the HFD-induced expansion of inguinal fat. Furthermore, Paton, Rogowski [30] found that LCN2 administration increases energy expenditure in vivo and up-regulates expression of genes participating in $\beta$ oxidation to increase fatty acid oxidation in adipocytes. Indeed, Zhang, $\mathrm{Wu}$ [2] stated that LCN2 administration to 3T3-L1 adipocytes has been shown to increase the expression of PPAR $\gamma$ and adiponectin and attenuate the inhibitory effect of TNF $\alpha$ on insulin-stimulated glucose uptake. T2DM is worldwide health problem. Its pathophysiology has been referred to the decrease in insulin secretion, increase in insulin resistance, and elevation of production of hepatic glucose [31]. Diet and exercise are important in treatment of diabetes. The results of our study declared that acute exercise decreased serum LCN2 which may be considered as a potential mechanism by which exercise increased insulin sensitivity and helping in treatment of T2DM.

\section{Conclusion}

Serum level of LCN2 is increased in prediabetic rats and this increase is deteriorated by an acute exercise indicating that LCN2 has a potential role in the occurrence of insulin resistance in T2DM. Also, we can conclude that acute exercise increased insulin sensitivity in prediabetic rats through decreasing serum LCN2. Moreover, it is concluded that LCN2 may be considered as one of the biomarkers used to assess the improvement of the insulin sensitivity.

\section{Acknowledgements}

We provide our sincere gratitude to Physiology Department, Faculty of Medicine, Zagazig University, Egypt, for supporting this study to be achieved.

\section{References}

[1] Yan, Q.W., et al., The adipokine lipocalin 2 is regulated by obesity and promotes insulin resistance. Diabetes, 2007. 56(10): p. 2533-40.

[2] Zhang, J., et al., The role of lipocalin 2 in the regulation of inflammation in adipocytes and macrophages. Mol Endocrinol, 2008. 22(6): p. 1416-26.

[3] Guo, H., et al., Lipocalin-2 deficiency impairs thermogenesis and potentiates diet-induced insulin resistance in mice. Diabetes, 2010. 59(6): p. 1376-85.

[4] Zhang, Y., et al., Lipocalin 2 expression and secretion is highly regulated by metabolic stress, cytokines, and nutrients in adipocytes. PLoS One, 2014. 9(5): p. e96997.

[5] Teixeira-Lemos, E., et al., Regular physical exercise training assists in preventing type 2 diabetes development: focus on its antioxidant and anti-inflammatory properties. Cardiovasc Diabetol, 2011. 10: p. 12.

[6] Conn, V.S., et al., Metabolic effects of interventions to increase exercise in adults with type 2 diabetes. Diabetologia, 2007. 50(5): p. $913-21$

[7] Saely, C.H., K. Geiger, and H. Drexel, Brown versus white adipose tissue: a mini-review. Gerontology, 2012. 58(1): p. 15-23.

[8] Kiess, W., et al., Adipocytes and adipose tissue. Best Pract Res Clin Endocrinol Metab, 2008. 22(1): p. 135-53.

[9] Seneff, S., G. Wainwright, and L. Mascitelli, Is the metabolic syndrome caused by a high fructose, and relatively low fat, low cholesterol diet? Arch Med Sci, 2011. 7(1): p. 8-20.

[10] Coelho, M., T. Oliveira, and R. Fernandes, Biochemistry of adipose tissue: an endocrine organ. Archives of Medical Science : AMS, 2013. 9(2): p. 191-200.

[11] Sun, K., C.M. Kusminski, and P.E. Scherer, Adipose tissue remodeling and obesity. The Journal of Clinical Investigation, 2011. 121(6): p. 2094-2101.

[12] Roupas, N.D., et al., The effect of prolonged aerobic exercise on serum adipokine levels during an ultra-marathon endurance race. Hormones (Athens), 2013. 12(2): p. 275-82.

[13] Guo, H., et al., Evidence for the Regulatory Role of Lipocalin 2 in High-Fat Diet-Induced Adipose Tissue Remodeling in Male Mice. Endocrinology, 2013. 154(10): p. 3525-3538.

[14] Alwahsh, S.M., et al., Diet high in fructose leads to an overexpression of lipocalin-2 in rat fatty liver. World $\mathrm{J}$ Gastroenterol, 2014. 20(7): p. 1807-21.

[15] Lopes, L.R., et al., Reduced inflammatory response in rats fed fat-rich diets: Role of leukotrienes. Life Sciences, 2000. 67(1): p. 13-21.

[16] Ropelle, E.R., et al., Acute exercise modulates the Foxo1/PGC-1 $\alpha$ pathway in the liver of diet-induced obesity rats. The Journal of Physiology, 2009. 587(9): p. 2069-2076.

[17] Nayak, Y. and V. Hillemane, Antidiabetic activity of benzopyrone analogues in nicotinamide-streptozotocin induced type 2 diabetes in rats. 2014. 2014: p. 854267. 
[18] Bonora, E., et al., Homeostasis model assessment closely mirrors the glucose clamp technique in the assessment of insulin sensitivity: studies in subjects with various degrees of glucose tolerance and insulin sensitivity. Diabetes Care, 2000. 23(1): p. 57-63.

[19] Catalan, V., et al., Increased adipose tissue expression of lipocalin-2 in obesity is related to inflammation and matrix metalloproteinase- 2 and metalloproteinase- 9 activities in humans. J Mol Med (Berl), 2009. 87(8): p. 803-13.

[20] Sommer, G., et al., Lipocalin-2 is induced by interleukin-1beta in murine adipocytes in vitro. J Cell Biochem, 2009. 106(1): $\mathrm{p}$. 103-8.

[21] Luciano, E., et al., Endurance training improves responsiveness to insulin and modulates insulin signal transduction through the phosphatidylinositol 3-kinase/Akt-1 pathway. Eur J Endocrinol, 2002. 147(1): p. 149-57.

[22] Aoi, W., et al., Effect of exercise on hepatic gene expression in rats: a microarray analysis. Life Sci, 2004. 75(26): p. 311728.

[23] Flores, M.B., et al., Exercise improves insulin and leptin sensitivity in hypothalamus of Wistar rats. Diabetes, 2006. 55(9): p. 2554-61.

[24] Pauli, J.R., et al., Acute physical exercise reverses Snitrosation of the insulin receptor, insulin receptor substrate 1 and protein kinase $\mathrm{B} / \mathrm{Akt}$ in diet-induced obese Wistar rats. J Physiol, 2008. 586(2): p. 659-71.
[25] Peres, S.B., et al., Endurance exercise training increases insulin responsiveness in isolated adipocytes through IRS/PI3kinase/Akt pathway. J Appl Physiol (1985), 2005. 98(3): p. 1037-43.

[26] Ropelle, E.R., et al., Reversal of diet-induced insulin resistance with a single bout of exercise in the rat: the role of PTP1B and IRS-1 serine phosphorylation. J Physiol, 2006. 577(Pt 3): p. 997-1007.

[27] Law, I.K., et al., Lipocalin-2 deficiency attenuates insulin resistance associated with aging and obesity. Diabetes, 2010. 59(4): p. 872-82.

[28] Jun, L.S., C.P. Siddall, and E.D. Rosen, A minor role for lipocalin 2 in high-fat diet-induced glucose intolerance. Am J Physiol Endocrinol Metab, 2011. 301(5): p. E825-35.

[29] Guo, H., et al., Evidence for the regulatory role of lipocalin 2 in high-fat diet-induced adipose tissue remodeling in male mice. Endocrinology, 2013. 154(10): p. 3525-38.

[30] Paton, C.M., et al., Lipocalin-2 increases fat oxidation in vitro and is correlated with energy expenditure in normal weight but not obese women. Obesity (Silver Spring), 2013. 21(12): p. E640-8.

[31] DeFronzo, R.A., From the Triumvirate to the Ominous Octet: A New Paradigm for the Treatment of Type 2 Diabetes Mellitus. Diabetes, 2009. 58(4): p. 773-795. 